\title{
¿Por qué, si tenemos el mismo nivel educativo, no ganamos lo mismo? Diferenciación Salarial en Santiago de Cali
}

\author{
If we Have the Same Educational Level, why do not we \\ Earn the Same? \\ Salary Differentiation in Santiago de Cali
}

\author{
Por que, se temos o mesmo nível educativo, não \\ ganhamos igual? \\ Diferenciação salarial em Santiago de Cali (Colômbia)
}

Diana Marcela Jiménez Restrepo ${ }^{1}$

Profesora titular Departamento de Economía de la Universidad del Valle, Cali, Colombia

diana.marcela.jimenez@correounivalle.edu.co

Anderson Pino Garcés ${ }^{2}$

Estudiante Maestría en Políticas Públicas de la Universidad del Valle, Cali, Colombia

anderson.pino@correounivalle.edu.co

$1 \quad$ Magíster en Economía Aplicada.

2 Economista. 
¿Por qué, si tenemos el mismo nivel educativo, no ganamos lo mismo? Diferenciación Salarial en Santiago de Cali

\section{Resumen}

El objetivo del presente artículo es calcular la diferencia salarial entre hombres y mujeres, en Cali, Colombia, teniendo en cuenta los años de educación. Para lograrlo, se creó y estimó el índice de diferencia salarial, usando los datos de la encuesta de Empleo y Calidad de Vida realizada por la Alcaldía de Cali y el Ministerio de Trabajo. Los resultados muestran que entre los ocupados de esta ciudad, independientemente del nivel educativo que presenten, las mujeres devengan un salario semanal inferior al de los hombres y solo en el caso de la secundaria, esta desigualdad se hace menor.

Palabras clave: economía laboral; salarios; capital humano; educación y empleo.

\section{Abstract}

The purpose of this paper is to calculate the salary difference between men and women, in Cali, Colombia, considering the educational level. In order to achieve this, a salary difference index was created and estimated, using data from the Employment and Quality of Life survey conducted by the Mayor's Office of Cali and the Ministry of Labor. The results show that, regardless of the educational level presented by the employed in this city, women earn a weekly salary lower than that of men; and only in the case of secondary school, this inequality becomes lower.

Keywords: labor economics; wage; human resources; education and employment.

\section{Resumo}

O objetivo deste artigo é calcular a diferença salarial entre homens e mulheres, controlando os anos de educação. Para isso, criamos e estimamos o índice de diferença salarial (IDS), usando os dados da enquete de "Empleo y Calidad de Vida", realizada pela Prefeitura da Cidade de Cali e o Ministério de Trabalho. Os resultados mostram que, dentro dos ocupados da cidade e independentemente do nível educativo, as mulheres ganham um salário semanal inferior do que os homens; apenas no caso do nível secundário, esta desigualdade é inferior.

Palavras-chave: economia do trabalho, salários, capital humano, educação e emprego.

¿Cómo citar este artículo? / How to quote this article?

Jiménez, D. M. \& Pino, A. ¿Por qué, si tenemos el mismo nivel educativo, no ganamos lo mismo? Diferenciación Salarial en Santiago de Cali. Sociedad y Economía, (35), 32-49. https://doi.org/10.25100/sye.voi35.7301 


\section{Introducción}

La hipótesis de la discriminación salarial es clara: por razones ajenas a las características que inciden en la productividad de los oferentes de trabajo, a unos se les reconoce un salario mayor. Estas razones, por lo general, tienen que ver con la condición de género de los trabajadores o con su reconocimiento étnico, de manera que las mujeres perciben alrededor de $20 \%$ menos de salario en relación con el recibido por un hombre que tenga tareas laborales similares. $\mathrm{El}$ anterior dato fue tomado del periódico $\mathrm{El}$ Tiempo (06/03/2015) siendo una muestra de que este tema ya no solo pertenece a las agendas académicas, desde donde ha sido ampliamente estudiado, sino que ya es reconocido como un problema dentro de las agendas sociales.

La segregación en el mercado laboral es también una hipótesis a la cual se recurre para explicar las diferencias en los salarios. De acuerdo con Kramar (1983), la segregación ocupacional causa efectos en los salarios relativos de hombres y mujeres ya que la sobrepoblación, en alguna ocupación, genera reducciones en los retornos a las cualificaciones de los trabajadores.

El problema que se intenta analizar con este ejercicio investigativo parte del hecho de que la igualdad de género por la que se aboga abarca la igualdad en el acceso a las distintas oportunidades de las dimensiones en la vida de los seres humanos: educación, salud, esparcimiento, seguridad, trabajo. Pero, y al respecto de esto último, en cuanto a la dimensión laboral, es bien sabido que, a pesar de que ha aumentado la participación femenina en el mercado laboral y a que las mujeres en Colombia tienen en promedio más años de educación que los hombres (ver Galvis, 2010; Hoyos, Ñopo y Peña, 2010; Piñeros, 2009) la desigualdad salarial entre hombres y mujeres no tiende a disminuir.

Pareciera entonces, como una de nuestras hipótesis, que los postulados de la teoría credencialista (Collins, 1989) no aplican y que entonces, hay aspectos, características de las personas que en el mercado laboral reciben distintas ponderaciones, que por lo general se construyen a partir de juicios de valor, puestos por encima de la productividad, la habilidad y del propio nivel educativo alcanzado. No obstante, no podemos quedarnos solo con la explicación discriminatoria y obviar el hecho de que las diferencias salariales bien pueden ser explicadas por razón de los distintos niveles de experiencia laboral o por diferentes jornadas laborales, pero, por lo pronto, esta investigación se enfocará en el análisis del nivel educativo alcanzado.

A pesar de que la igualdad salarial entre hombres y mujeres debe pasar a lo real y dejar de ser parte del discurso, se quiere, con este ejercicio, insistir en el análisis de la diferenciación controlado solo por el nivel educativo alcanzado; este último, tomado como una variable que nos aproxime a la productividad de cada persona. Así, nuestro objetivo principal es el de calcular una medida que nos dé cuenta de la diferenciación salarial por sexo en la ciudad de Cali.

Para alcanzar el objetivo principal de esta investigación, como metodología se propone un nuevo índice de diferenciación salarial, condicionado al nivel educativo, controlando así, por una aproximación al nivel de productividad de cada persona. Se usan los datos de la Encuesta de Empleo y Calidad de Vida de Cali (2012:11 2013:01), aplicada por la Alcaldía de Cali y el Ministerio de Trabajo, aprovechando que han sido pocos los trabajos que se han hecho circular en el ambiente académico, en los cuales se use la fuente de información que aquí se menciona. De paso, con este ejercicio de investigación también se quiere mostrar el interés y abogar por que se continúe con este tipo de encuestas que facilitan los análisis microlocales dejando a un lado la generalidad que imponen las encuestas nacionales de hogares que carecen del detalle de las particularidades de la ciudad.

Este documento sigue con una discusión sobre la manera como se ha abordado el tema que aquí nos llama, mencionando algunos documentos, tanto nacionales como internacionales, del gran número de literatura que puede leerse y en la que se ha discutido el tema de la diferenciación salarial. Aprovechando dicha 
¿Por qué, si tenemos el mismo nivel educativo, no ganamos lo mismo?

discusión, se incluyen los planteamientos teóricos que sustentan el tema central. Luego, en la tercera sección, se presenta el índice con el cual se medirá la desigualdad salarial en la ciudad de Cali para, a partir de la cuarta sección, presentar el análisis empírico: estadísticas descriptivas y resultados. Por último, las conclusiones de este ejercicio investigativo.

\section{Diferenciación salarial: ¿Qué se sabe?}

El indicador general para medir la desigualdad o la diferencia de los ingresos laborales entre hombres y mujeres es llamado brecha salarial. Con este se calcula la diferencia entre los salarios promedios de hombres y mujeres como proporción de los ingresos de los hombres. Dicho cálculo bien puede realizarse teniendo en cuenta las remuneraciones por hora, por semana, al mes e incluso, anual.

Según el informe Igualdad salarial: Guía introductoria de la OIT (2013), la tendencia mundial de la brecha salarial por género muestra, por un lado, que las mujeres ganan alrededor del $77 \%$ del salario de los hombres y de otro, que hay mayor igualdad salarial en el sector público que en el privado. De hecho, la diferencia en la remuneración de hombres y mujeres tiene determinantes que también la hacen variar dependiendo del país que se analice, así como de la ocupación estudiada.

El listado de las causas de la desigualdad salarial es amplio, social y académicamente reconocido. Bien se puede comenzar con mencionar las diferencias en educación o formación entre hombres y mujeres, a pesar de que los logros educativos de estas últimas han ido en aumento, a tal punto, que en promedio pueden tener mayores niveles educativos que los hombres. Otra fuente es la experiencia laboral, la cual suele ser mayor en los hombres, ya que estos no interrumpen su vida laboral por responsabilidades en el hogar, como lo es la maternidad; sin embargo, para el caso de Colombia, Pérez (2016) encontró que no hay evidencia del efecto opting out -exclusión- en el mercado laboral, cuando las mujeres se convierten en madres. Las jornadas laborales, según el tipo de contratación, que determinan empleos a tiempo parcial o completo, también causan las diferencias salariales entre hombres y mujeres una vez que los empleos de tiempo parcial suelen ser cubiertos, en una amplia mayoría, por mujeres. De otro lado, las empresas de menor tamaño, que suelen ofrecer salarios más bajos que las empresas grandes, prefieren contratar más mujeres que hombres.

Las anteriores causas listadas se pueden agrupar en dos grandes razones para explicar la diferenciación salarial: una de ellas, la segregación laboral y la otra, la discriminación en el mercado laboral.

La segregación laboral es el resultado de elegir cierto tipo de ocupaciones al encontrar características afines en ellas. Un ejemplo es el que las mujeres tiendan por ocupaciones que implican destrezas motrices finas, como lo son las actividades de confección, el cuidado de personas, mientras los hombres tienden a ocuparse en trabajos de mayor exigencia física. Esta elección individual, está permeada, sin duda, por la existencia de estereotipos que sesgan la decisión de hombres y mujeres a la hora de elegir una ocupación o un empleo. No obstante, entendida la segregación como consecuencia de una elección individual, pareciera no nociva o sin efectos negativos sobre la situación laboral de las personas, sin embargo, son los resultados de la segregación los que sí muestran sus impactos negativos a nivel laboral: ¿Qué pasa con el salario de una ocupación específica cuando hay un exceso de oferentes de trabajo dispuestos a ocuparse en ella? La saturación, el exceso de oferta, lo que lleva es a disminuir el precio del factor trabajo, es decir, a salarios bajos.

La segregación horizontal profesional hace referencia a la imposición tanto social como individual, de un restringido abanico de alternativas de sectores de la economía y de puestos de trabajo para buscar un empleo. En el caso específico de las mujeres, este tipo de segregación surge de las decisiones de emplearse en sectores 
y ocupaciones que suelen ser reconocidas como las idóneas para las mujeres que, por lo general, reportan bajos salarios. De hecho, la segregación vertical profesional tiene que ver con que las mujeres tienen una escasa representación en los niveles salariales más altos e incluso en las mejores posiciones laborales. $\mathrm{Al}$ respecto Chávez y Ríos (2014) señalan que, para las siete principales áreas metropolitanas de Colombia, existe una barrera invisible que les impide a las mujeres acceder a cargos jerárquicos (techo de cristal). Con esto, una consecuencia de la segregación laboral es la inminente diferencia salarial entre hombres y mujeres.

Una de las principales razones a la que se recurre para explicar la diferencia salarial, es el enfoque de la discriminación laboral. Esta se entiende como el trato diferencial que recibe un grupo de personas por razones diferentes a su productividad laboral, por ejemplo, por características observables como su condición de género o de procedencia étnica.

Cuando el empleador forma un juicio de valor a partir de las características observables (sexo, género, edad, etnia, nivel socioeconómico, entre otras) de los potenciales trabajadores, para superar la incertidumbre acerca de las habilidades y de la productividad de estos, el tipo de discriminación que se está llevando a cabo es la estadística (Phelps, 1972; Arrow, 1973). Un ejemplo en este caso es generalizar que todas las mujeres presentan menor productividad laboral por el hecho de ser mujeres o que los aspirantes a un empleo, entendidos estos como quienes están en búsqueda de su primera plaza laboral, presentan menores niveles de productividad laboral que personas mayores, ya que entre estos existe un diferencial en experiencia laboral. Si, por el contrario, los empleadores están dispuestos a pagar para no interactuar con personas de un grupo específico (con características físicas, socioeconómicas u otras, homogéneas entre ellos), la discriminación está basada en gustos o preferencias (Becker, 1957).

Para Colombia existe un análisis de diferenciación salarial focalizado en el sector formal, en el cual Iregui, Melo y Ramírez (2011) muestran que existen diferencia en los salarios cuando se comparan los sectores económicos, las firmas y las categorías ocupacionales, concluyendo que los sectores con mayor productividad laboral, mayor relación capital trabajo, ofrecen salarios más altos mientras que en términos de las categorías ocupacionales, los directivos son quienes devengan los mayores salarios en las compañías. Aunque ambos resultados eran de esperarse, resaltamos el llamado que hacen estas autores a la manera como se ha abordado este tema, señalando los autores que se han encargado de hacerlo.

\section{Discriminación laboral versus segregación laboral: el enfoque para explicar las brechas salariales}

Los análisis de la economía laboral sobre la segregación se han concentrado en estudiar y cuantificar la participación de hombres y mujeres en ocupaciones específicas. Para ello, se estiman diversos índices: de concentración, segregación, representación y distribución (Nicolás, López y Riquelme, 2009) siendo los índices de desigualdad o de disimilitud de Duncan y Duncan (1955) o el de Karmel y Maclachlan (1988) de los más usados. Estos indicadores, al mostrar el grado de concentración o de saturación de mujeres o de hombres en algún tipo de empleo, también son usados para analizar las diferencias salariales que se originan ante el exceso de mujeres en una ocupación frente a una menor participación de hombres en esa misma labor (Kramar, 1983; Jarman, Blackburn y Racko, 2012).

Pero es el enfoque de la discriminación laboral con el que mayoritariamente se estudia la diferencia salarial. En primer lugar y por la cantidad de investigaciones que se han realizado, se encuentran los estudios entre sexos o bien definiendo dos submuestras de individuos en las cuales se quiera estudiar la brecha salarial entre agrupaciones de personas, por ejemplo, personas de etnias distintas o de diferentes países de procedencia (Wellington, 1993; 1994; Rodgers, 2006). 
La metodología Blinder-Oaxaca (BO) es la estándar para cuantificar las diferencias salariales fruto de la discriminación laboral. Esta metodología está basada en la estimación de ecuaciones mincerianas, para grupos de individuos, donde se tienen en cuenta características de dotación de capital humano (nivel educativo, experiencia laboral) como determinantes de la brecha salarial o también permite concluir si las diferencias salariales se deben a factores que no se han incluido en la ecuación y por tanto hay evidencia de discriminación.

Para el caso colombiano, son varios los estudios en los que se ha empleado la metodología BO para determinar si las brechas salariales se deben a diferencias en el capital humano o por razones de discriminación. El primer ejercicio para Colombia fue el de Tenjo (1993) quien encontró que los retornos educativos fueron superiores para hombres, durante la década de 1980, y que la diferencia salarial entre hombres y mujeres se debió por motivos de discriminación y no por diferencias en capital humano. Algunos de los tantos trabajos que utilizan la metodología BO, con datos de Colombia, son los de Baquero (2001), Bernat (2005; 2007), Tenjo y Herrera (2009), Galvis (2010), diferenciándose en la muestra empleada y en la afinación de la técnica de estimación al emplear regresiones por cuantiles o curvas de discriminación, por ejemplo. Recientemente, Cortés y Flórez (2016) muestran que en el departamento de Santander, entre $25 \%$ y $30 \%$ de la diferencia salarial entre hombres y mujeres se debe a un componente discriminatorio no explicado.

Para el ejercicio que aquí nos ocupa, no estamos pensando en usar la metodología Blinder-Oaxaca porque estamos proponiendo nuestro propio indicador de diferencia salarial, controlando por nivel educativo sin que nos permita validar la hipótesis de discriminación laboral como la causante de la brecha entre los salarios. De hecho, para una investigación futura e intentando ser más rigurosos, queremos analizar, con mayor exactitud, otras características laborales como por ejemplo, un análisis de diferenciación salarial por puesto de trabajo, por ocupación o igual número de años de experiencia laboral, no obstante, algunas de las estadísticas descriptivas que se expondrán, muestran los casos de diferencia salarial controlando por estas variables.

Como esperamos que la inequidad o desigualdad salarial se deba a diferencias educativas, de cualificaciones, habilidades o destrezas, proponemos el siguiente índice de diferenciación salarial.

\section{3. ¿Cuánto debe ganar de más, una mujer o un hombre, para que exista igualdad salarial? Un índice de diferenciación salarial controlando por nivel educativo}

El índice con el cual cuantificaremos la desigualdad salarial tiene la forma:

$$
\text { IDS }=\left[1-\left(\frac{W_{m}}{W_{h}}\right)\right][1]
$$

Donde, $W_{i}$ es el salario/periodo promedio para i: Mujeres, Hombres.

De manera que en la razón $\left(\frac{W_{m}}{W_{h}}\right)$ estamos midiendo la brecha salarial (OIT, 2013). Para este ejercicio, el periodo escogido es la semana.

Teorema 1. En condiciones de igualdad, la brecha salarial $\left(\frac{W_{m}}{W_{h}}\right)$ es exactamente igual a 1 .

Cuando el salario de una mujer es proporcionalmente el $100 \%$ del salario del hombre, la diferenciación salarial entre estos es igual a cero, IDS $=0$.

$$
\begin{gathered}
\text { Para } \\
W_{m}=W_{h} \Leftrightarrow \frac{W_{m}}{W_{h}}=1 \Rightarrow I D S=\left[1-\left(\frac{W_{m}}{W_{h}}\right)\right]=0[2]
\end{gathered}
$$

Teorema 2. En condiciones de desigualdad salarial a favor de las mujeres, la brecha salarial es mayor a 1 . 
Cuando la mujer devenga un salario mayor al del hombre, el IDS tomará valores negativos. Luego, para los hombres debe aumentar en |IDS| veces su ingreso laboral para que se logre la igualdad salarial.

Para

$W_{m}>W_{h} \Leftrightarrow \frac{W_{m}}{W_{h}}>1 \Rightarrow I D S=\left[1-\left(\frac{W_{m}}{W_{h}}\right)\right]<0[3]$

Teorema 3. En condiciones de desigualdad salarial a favor de los hombres, la brecha salarial es menor a 1 .

Cuando el hombre tiene un asalario mayor al de la mujer, el IDS tomará valores positivos. El ingreso laboral del hombre debe disminuir en IDS veces para que se logre la igualdad salarial.

$W_{m}<W_{h} \Leftrightarrow \frac{W_{m}}{W_{h}}<1 \Rightarrow I D S=\left[1-\left(\frac{W_{m}}{W_{h}}\right)\right]>0[4]$

Nótese que para los casos en los que el IDS arroja desigualdad en los salarios entre hombres y mujeres, se interpreta el cambio en el salario del hombre, que es la base en el componente de brecha $\left(\frac{W_{m}}{W_{h}}\right)$. De paso, este índice tiene la ventaja que bien puede usarse utilizando otra variable de control y arrojar resultados para lo que fue creado.

\section{4. ¿Qué tan diferentes son los salarios de hombres y mujeres en la ciudad de Cali?}

En el ejercicio empírico que aquí desarrollamos se hace uso de los datos en la encuesta de Empleo y Calidad de Vida para Santiago de Cali, realizada por el Ministerio de Trabajo y la Alcaldía de Cali entre los meses de noviembre de 2012 y enero de 2013. De acuerdo con la muestra de datos se puede clasificar, para el período en referencia, un total de 11.211 personas ocupadas en el mercado laboral de la ciudad, de las cuales $44 \%$ fueron mujeres, $78 \%$ no se autorreconocieron como afrocolombianos y $75 \%$ contó con estudios de secundaria (Gráfico 1).

Gráfico 1. Ocupados: hombres y mujeres con su respectivo nivel educativo

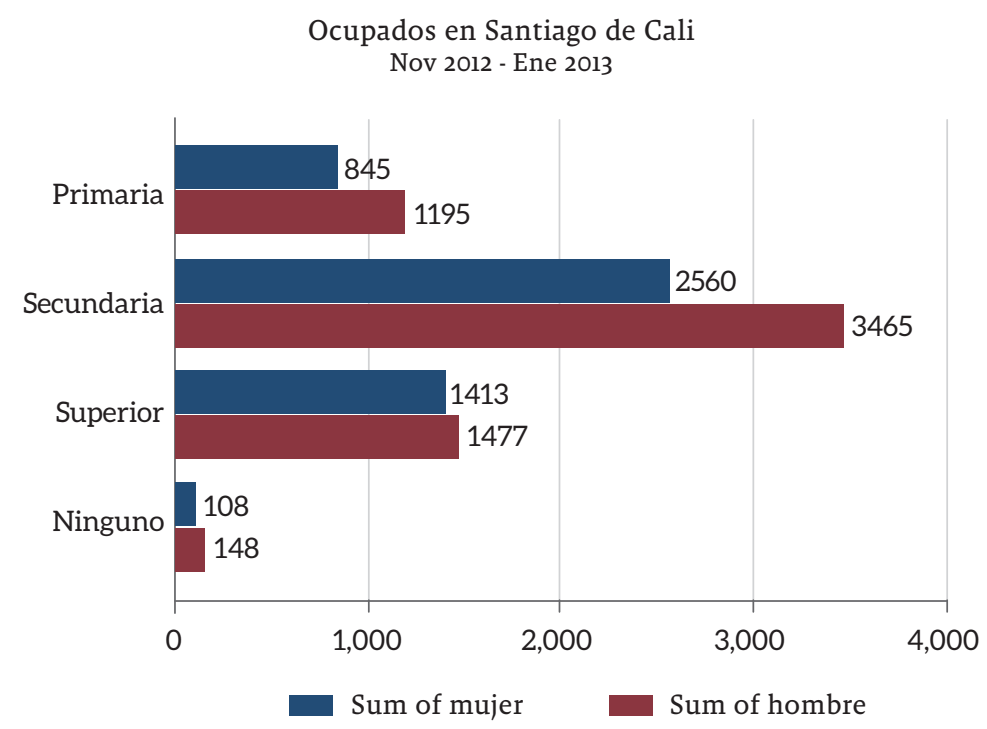

Fuente: cálculos propios con información de la EECV - Alcaldía de Cali y Mintrabajo (2013). 
En cuanto a las condiciones laborales de estos ocupados, el porcentaje entre quienes tenían contratos laborales y quienes no, estuvo repartido en partes iguales, lo cual muestra la precariedad laboral; esto luego se reafirmó con el hecho de que alrededor de 54,3\% de los ocupados devengaron hasta un salario mínimo legal vigente (S.M.L.V.), esto es, menos de $\$ 566.000$ mientras solo $13,6 \%$ tuvieron ingresos laborales superiores a dos S.M.L.V. Como puede apreciarse en el Gráfico 2, las mayores diferencias salariales las podemos empezar a distinguir entre los hombres y las mujeres que tuvieron jornadas laborales por fuera de las horas semanales que establece la Ley; estas diferencias, a favor de los hombres, se presentan tanto en el caso en el que se contaba con un contrato laboral como ante la ausencia del mismo.

Teniendo en cuenta dos elementos del capital humano, como lo son los años de educación y la experiencia laboral potencial, se deduce también un diferencial entre hombres y mujeres, nuevamente, a favor de ellos. La rentabilidad de la educación y de la experiencia laboral, además de ser más alta para los hombres, crece a una velocidad mayor respecto al caso para las mujeres, algo que bien puede notarse en ambos diagramas de dispersión, gracias a la mayor pendiente en la recta de ajuste para los hombres. Con esto, no solo hay indicios de diferencias en los ingresos laborales recibidos entre hombres y mujeres sino, además, discriminación laboral ya que, para un mismo nivel educativo, las mujeres devengan un salario inferior que el ganado por los hombres (Gráfico 3).

Respecto de los resultados hasta ahora encontrados, en términos del promedio de años de educación alcanzados (secundaria) y de las diferencias salariales a favor de los hombres, se mantienen los hallazgos reportados en el estudio de Tenjo (1993). Sin embargo, vale la pena mencionar, que estos resultados están condicionados a la generalidad de un análisis que no tiene en cuenta las características del puesto de trabajo o del tipo de ocupación. Es decir, ¿por qué, hombres y mujeres con igual número de años de educación, presentan distintos niveles de remuneración? A pesar de que se espera que las desigualdades en los ingresos laborales se deban a las diferencias educativas, la intuición

Gráfico 2. Promedio salarial para hombres y mujeres según la jornada laboral y la tenencia de contrato laboral

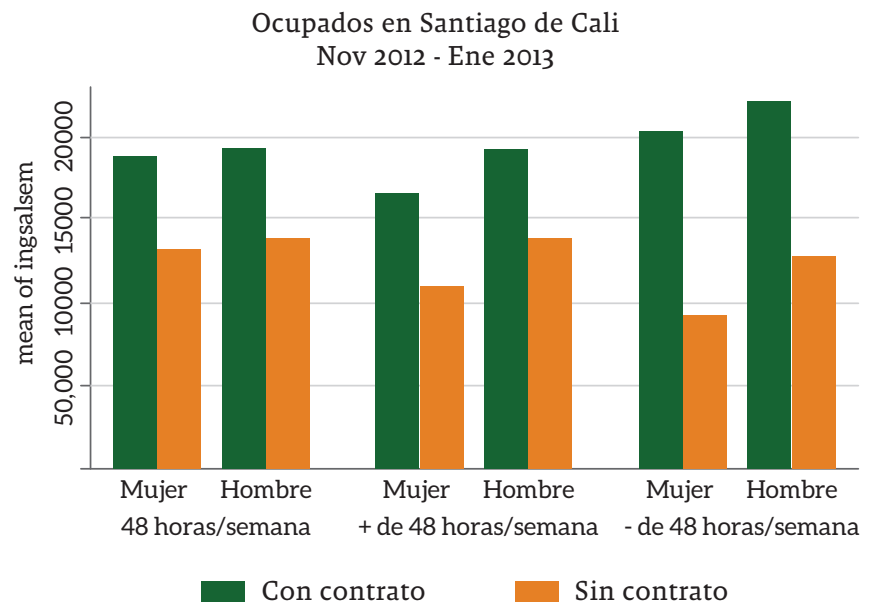

Fuente: cálculos propios con información de la EECV - Alcaldía de Cali y Mintrabajo (2013). 
Gráfico 3. Rentabilidad de la educación y de la experiencia laboral (potencial)

\section{Ocupados en Santiago de Cali Nov 2012 - Ene 2013}
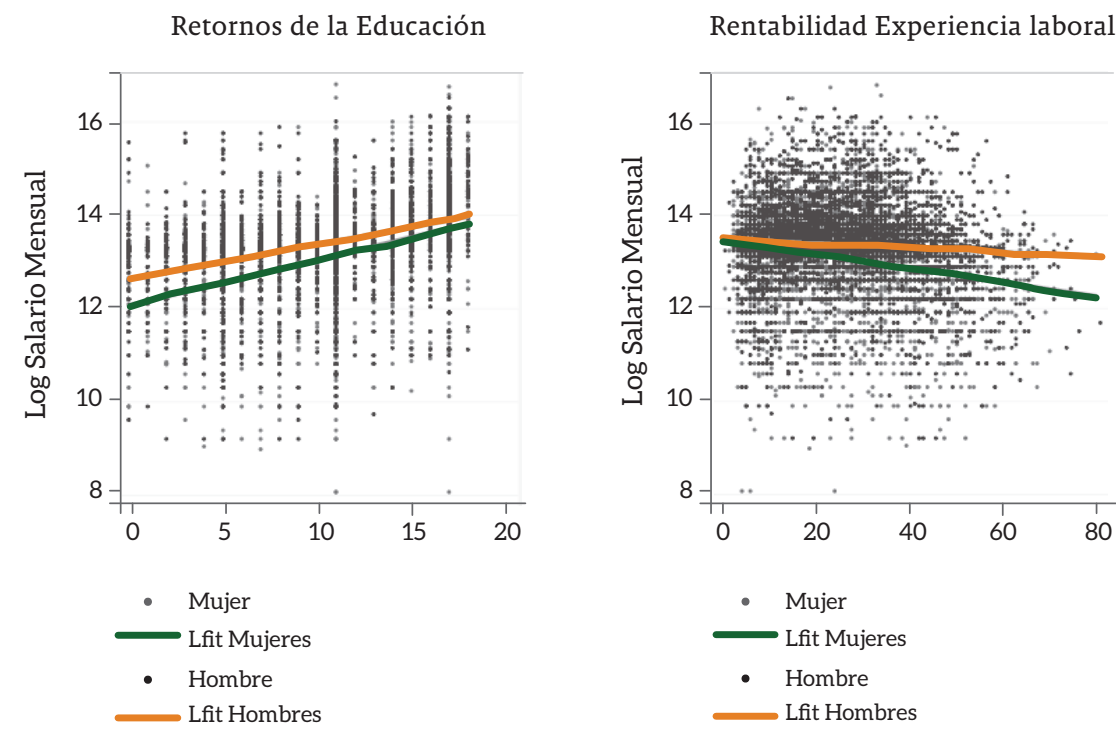

Fuente: cálculos propios con información de la EECV - Alcaldía de Cali y Mintrabajo (2013).

nos lleva a reafirmar que hay habilidades y destrezas que se adquieren o se perfeccionan en el aprender haciendo, con la experiencia laboral, en lo que por supuesto tiene mucho que ver el tipo de ocupación que se lleve a cabo.

Tomando entonces la variable tipo de empleado como una aproximación a la ocupación, tenemos que, en promedio, tanto para hombres como para mujeres, los mejores salarios mensuales están entre las categorías de empleado del gobierno o empleador, este último como bien puede esperarse (Gráfico 4). En estas anteriores categorías, se puede apreciar que la diferencia salarial está levemente a favor de las mujeres mientras que en el resto de las categorías para tipo de empleado, los hombres devengan en promedio, un salario mayor que las mujeres; como ejemplo de esto último, mírese el caso de trabajadores del servicio doméstico, en el que los hombres perciben salarios promedios de más de $\$ 500.000$ mientras que para las mujeres es menor el ingreso laboral promedio, lo que puede ser entonces, consecuencia de la segregación de las mujeres en este tipo de empleos.

La ciudad de Santiago de Cali está divida en 22 comunas entre las cuales albergan un total de 336 barrios, generando cinco zonas de la ciudad; en la zona sur de Cali están ubicadas las comunas $10,17,18,19,20$ y 22 . La zona oriente, con las comunas 11,12 y 16. El Distrito de Aguablanca, conformado por las comunas 13, 14, 15 y 21. En el nororiente de Cali están las comunas 4, 5, 6, 7 y 8 mientras el noroccidente cuenta con las comunas 1, 2, 3 y 9. A excepción del Distrito de Aguablanca, en el resto de las zonas se pueden encontrar comunas conformadas por barrios tanto de bajo como de alto estrato socioeconómico. En la zona sur, por ejemplo, está el barrio Siloé, de la comuna 20 mientras en la comuna 22 está 
Gráfico 4. Salario mensual promedio según tipo de empleado

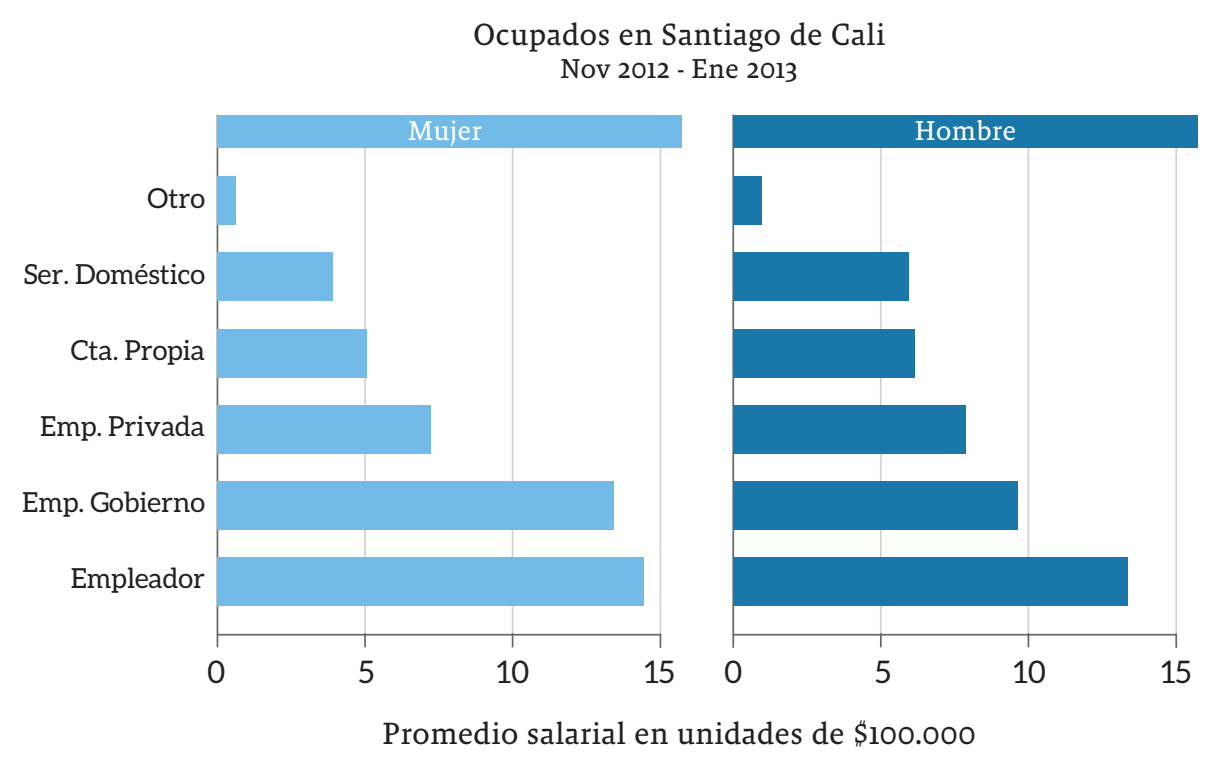

Fuente: cálculos propios con información de la EECV - Alcaldía de Cali y Mintrabajo (2013).

el sector de Ciudad Jardín; el primero de estos, Siloé, es uno de los barrios de la ciudad con mayor número de problemáticas socioeconómicas, a diferencia de Ciudad Jardín, en la comuna 22, donde la moda, a nivel de estratificación socioeconómica, es el estrato alto.

Para efectos del ejercicio práctico que aquí se presenta, usamos la división territorial de las comunas sin mayor intención que la de estudiar la diferenciación salarial al interior de las mismas sin proponer hipótesis al respecto pues, así como el ejemplo de Siloé y Ciudad Jardín podrían listarse otros casos para reafirmar la distribución espacial tan heterogénea de la estratificación socioeconómica en Cali. Aunque sabemos que se espera encontrar mayores niveles educativos, mejores salarios en las personas que habitan los barrios de estratos más altos, debido a la heterogeneidad que ya mencionamos, no vamos a realizar comparaciones entre comunas y aun así, tampoco al interior de estas, pues implicaría hacer un ejercicio más exhaustivo de las características sociales, económicas y laborales para ahondar en los condicionantes de la desigualdad salarial, análisis que dejamos para futuras investigaciones y que nos llevan a concentrarnos en la diferencia salarial condicionando por nivel educativo.

$\mathrm{Al}$ tener en cuenta el nivel educativo, como control o condicionamiento de la diferenciación salarial, asumimos que las personas con niveles educativos similares deberían presentar rentabilidades educativas no muy diferentes. Por lo pronto, podemos decir que existe un patrón claro al comparar los años de educación de hombres y mujeres, estando estas en ventaja frente a los años de educación promedio que presentan los hombres. En cambio, cuando se analiza el salario semanal promedio, lo que se visualiza es que, mayoritariamente, los hombres devengan un mayor salario que las mujeres (Gráfico 5).

De hecho, la regularidad de los resultados hallados con el IDS aquí propuesto es que la diferenciación salarial está a favor de los hombres. Para el caso de la categoría Ninguno dentro del nivel educativo, en el $65 \%$ de los casos, es decir, 
Gráfico 5. Promedio años de educación y promedio ingreso laboral semanal

Ocupados en Santiago de Cali

Nov 2012 - Ene 2013
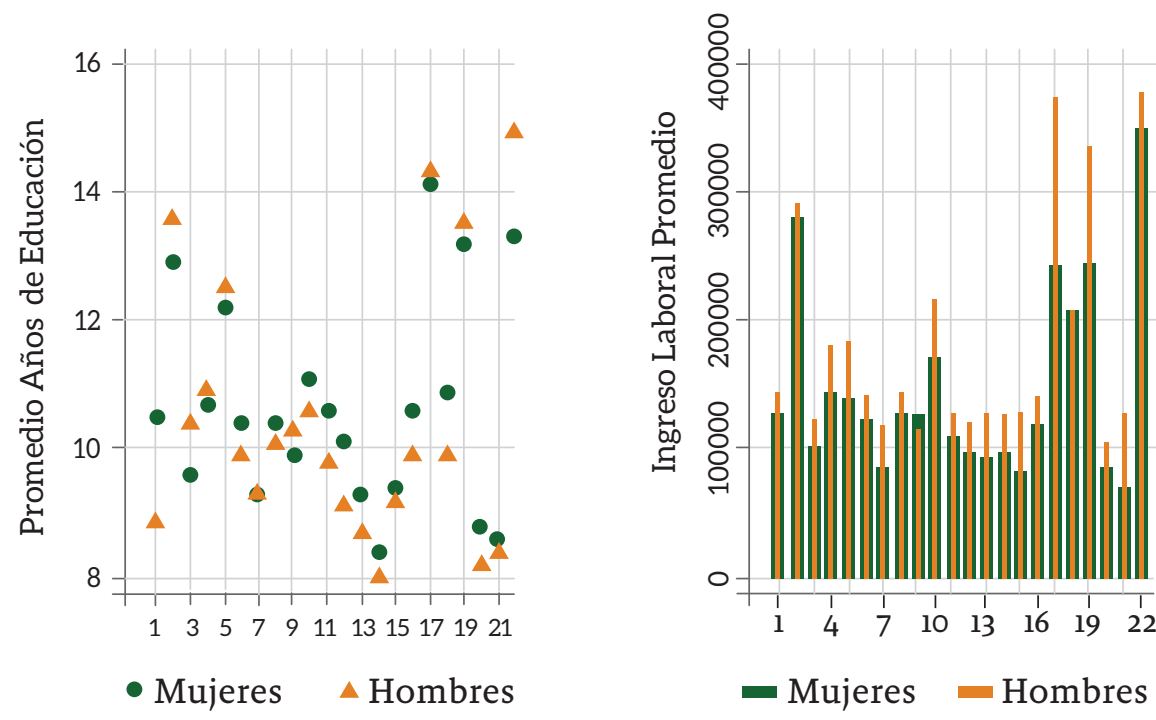

Fuente: cálculos propios con información de la EECV - Alcaldía de Cali y Mintrabajo (2013). Ingreso laboral en miles de pesos.

en 13 de las 22 comunas, el salario de las mujeres debería aumentar para que se alcanzara la igualdad salarial. Dentro de esta categoría, la diferencia salarial semanal promedio está alrededor de \$79,000 (Tabla 1).

La persistencia de la ventaja de los hombres frente a las mujeres en cuanto al ingreso laboral devengado a la semana se mantiene en el nivel educativo de primaria, tanto así que en $88 \%$ de los casos que corresponde a 17 comunas de la ciudad, el salario de las mujeres debería aumentar para que sea igual al ganado por los hombres. Para este nivel educativo, tenemos dos casos para los cuales el IDS es cercano a cero, lo cual muestra que la diferencia salarial semanal promedio entre hombres y mujeres es muy pequeña en términos relativos con los demás casos. En general, la diferencia salarial semanal promedio entre hombres y mujeres que tienen primaria es de $\$ 58.000$ (Tabla 2 ).
Para el nivel educativo de secundaria, solo en $9 \%$ de los casos, esto es en las comunas 2 y 9, debería aumentar el salario semanal que devengan los hombres para que hubiese igualdad de ingresos laborales, en ese orden de ideas, nuevamente, las mujeres con secundaria están devengando salarios menores al de los hombres con igual nivel educativo. Para este nivel, el promedio de la diferencia salarial semanal está en \$37.000 que, aunque continúa favoreciendo a los hombres, es inferior tanto para el caso de los niveles educativos ninguno y primaria (Tabla 3).

Para el nivel educativo más alto, el de superior, la diferencia promedio en los salarios semanales devengados entre hombres y mujeres, es de $\$ 70.000$, aproximadamente. De nuevo, solo en $14 \%$ de las veces, el ingreso laboral semanal medio de los hombres debería aumentar para que existiera igualdad salarial entre estos y las mujeres (Tabla 4). 
¿Por qué, si tenemos el mismo nivel educativo, no ganamos lo mismo? Diferenciación Salarial en Santiago de Cali

Tabla 1. Índice de diferenciación salarial para ninguno como nivel educativo

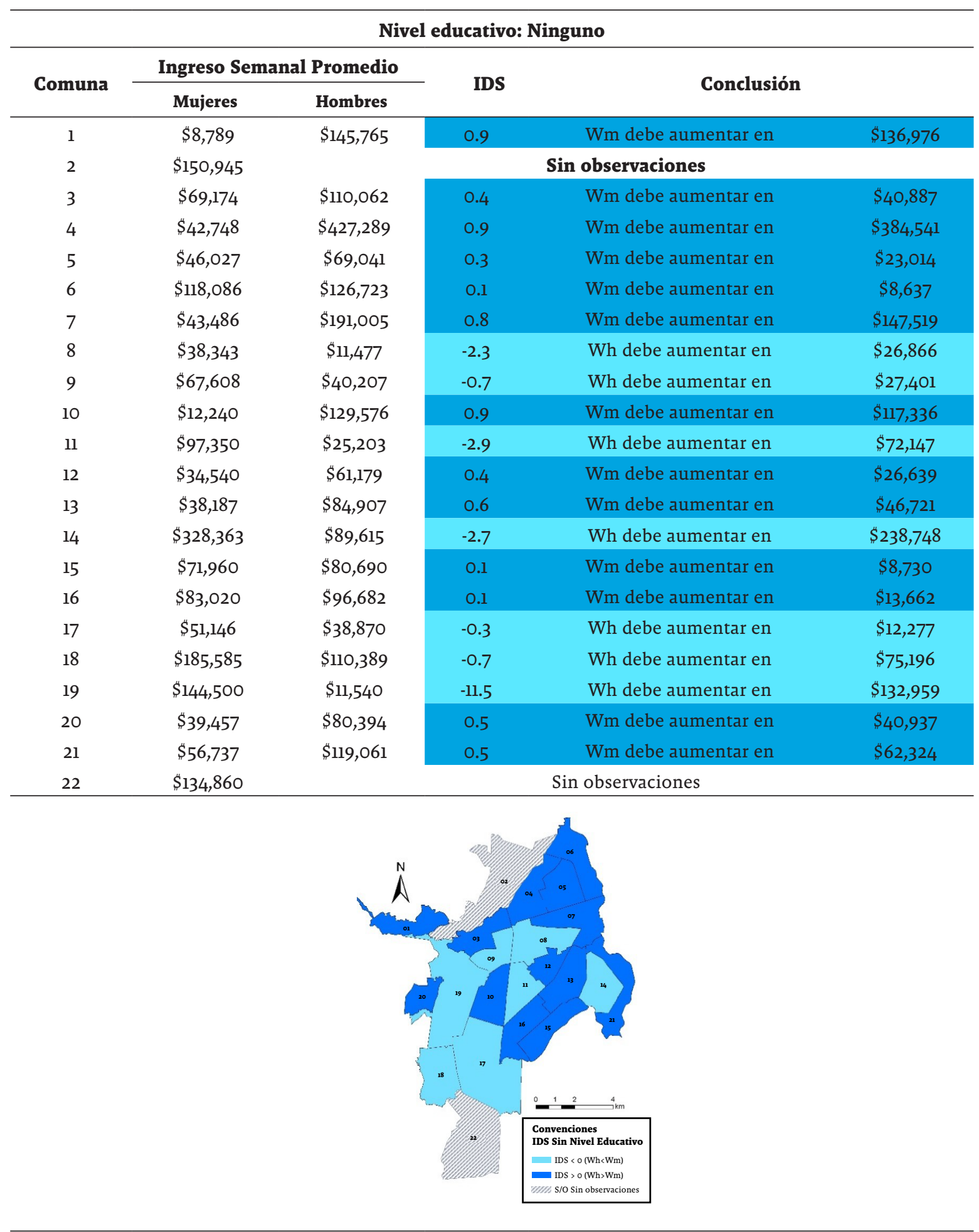

Fuente: cálculos propios con información de la EECV - Mintrabajo y Alcaldía de Cali 


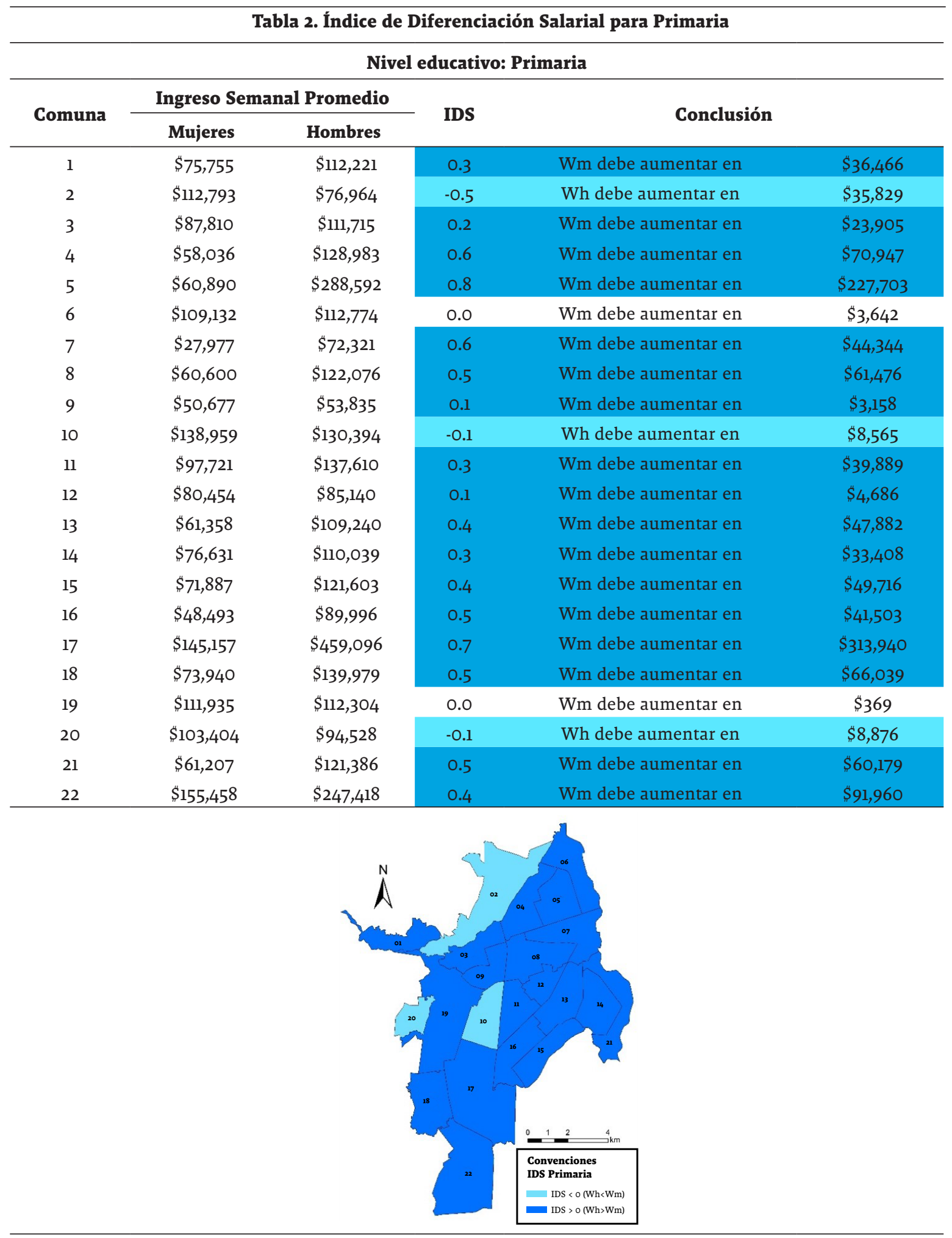

Fuente: cálculos propios con información de la EECV - Alcaldía de Cali y Mintrabajo (2013) 
¿Por qué, si tenemos el mismo nivel educativo, no ganamos lo mismo? Diferenciación Salarial en Santiago de Cali

Tabla 3. Índice de diferenciación salarial para secundaria

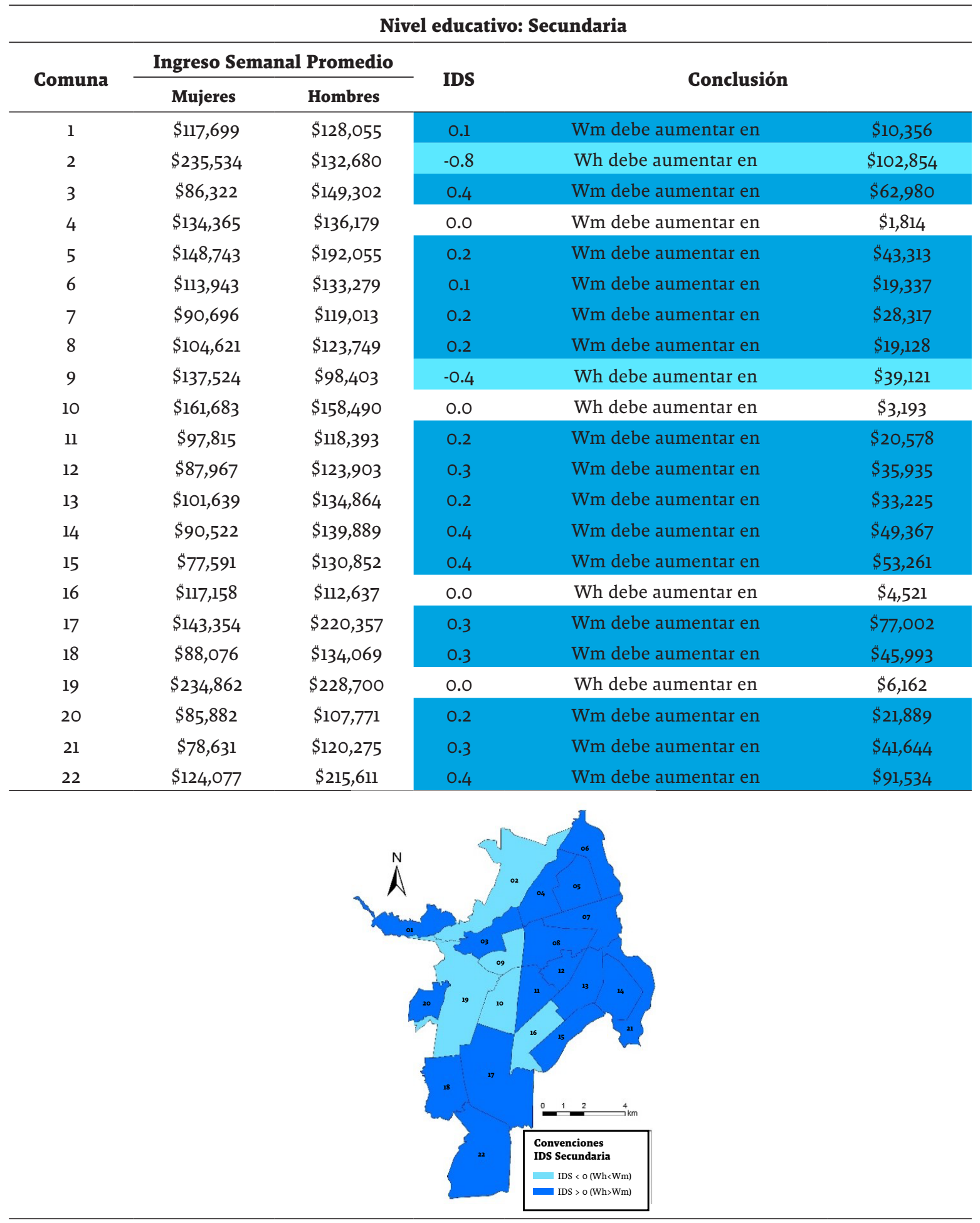

Fuente: cálculos propios con información de la EECV - Alcaldía de Cali y Mintrabajo (2013). 
Tabla 4. Índice de diferenciación salarial para superior

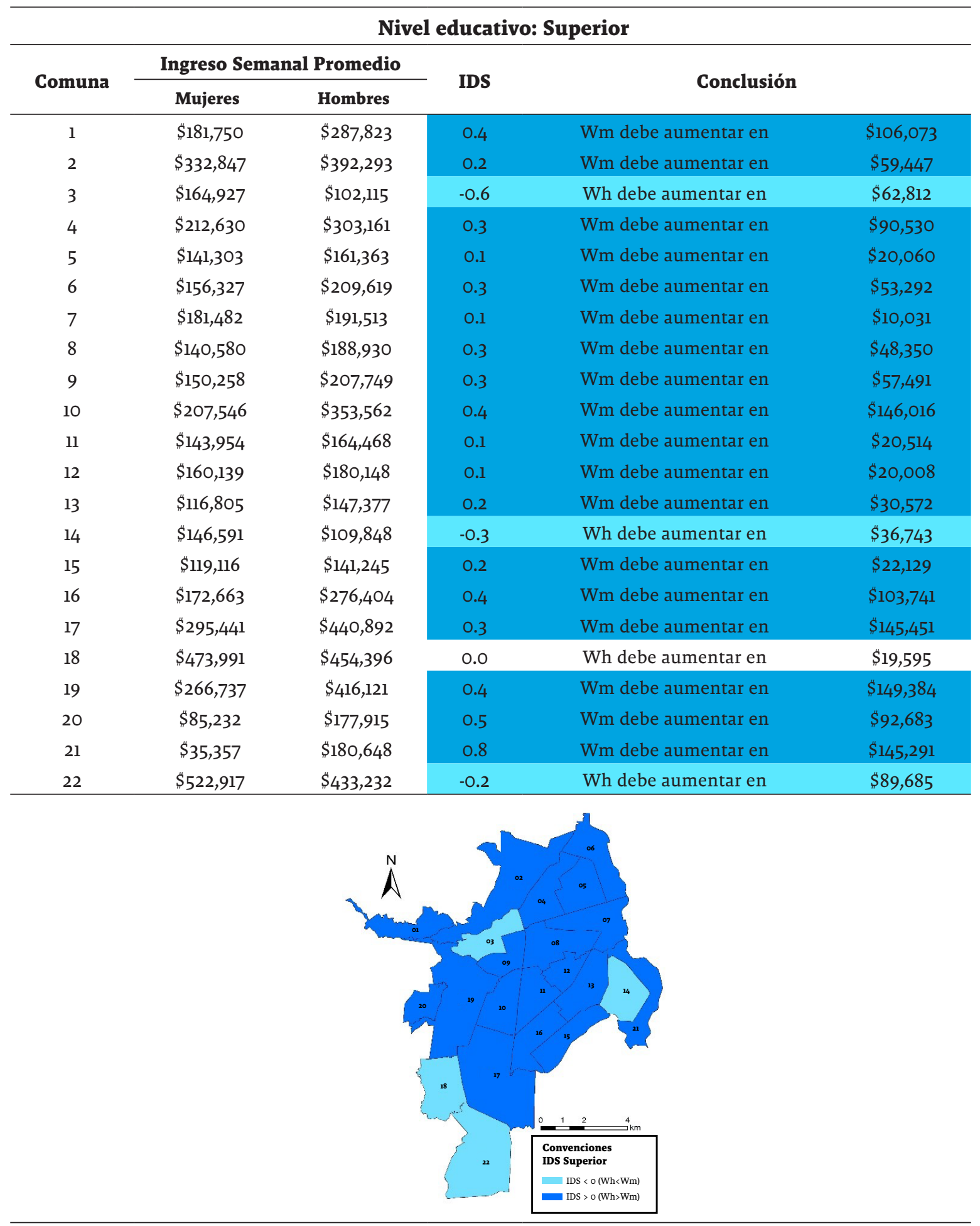

Fuente: cálculos propios con información de la EECV - Alcaldía de Cali y Mintrabajo (2013). 
¿Por qué, si tenemos el mismo nivel educativo, no ganamos lo mismo? Diferenciación Salarial en Santiago de Cali

\section{Conclusiones}

De acuerdo con los resultados aquí encontrados al estimar el IDS que se propone, con el cual estamos controlando por nivel educativo, se mantiene la tendencia de que la diferencia salarial favorece a los hombres. A pesar de que las mujeres tengan el mismo nivel educativo que los hombres e incluso más años de educación, los hombres ocupados en la ciudad de Cali devengan, en promedio, salarios semanales más altos. No obstante, esta diferencia es menor cuando se controla por el nivel educativo de secundaria que para el resto de categorías educativas; en el nivel superior está la mayor diferencia salarial a favor de los hombres.

Del análisis en las comunas se infieren los mismos resultados. A pesar de que las mujeres, en la gran mayoría de las comunas presentan más años de educación que los hombres, el salario es mayor para ellos, a excepción del caso de la comuna 9.

Se tendría que calcular este IDS para evaluar la diferencia salarial entre hombres y mujeres en un escenario mucho más preciso, como es el caso de la ocupación o el tipo de trabajo, controlando también por nivel educativo y hasta por la misma experiencia laboral para poder concluir la existencia de discriminación en el mercado laboral en la ciudad de Santiago de Cali pero, por lo pronto, sí podemos decir que existe desigualdad entre los salarios devengados por hombres y mujeres a pesar de que estos presenten el mismo nivel educativo.

\section{Referencias}

Alcaldía de Cali y Ministerio de Trabajo. (2013). Encuesta de Empleo y Calidad de Vida. Santiago de Cali, Colombia: Alcaldía de Cali, Ministerio de Trabajo.

Arrow, K. (1973). The Theory of Discrimination. En O. Ashenfelter y A. Rees (Eds.), Discrimination in Labor Markets (pp. 3-33). Nueva Jersey, Estados Unidos: Princeton University Press. Recuperado de http://www.jstor.org/stable/j.ctt13xiohs.5

Baquero, J. (2001). Estimación de la Discriminación Salarial por Género para los trabajadores asalariados urbanos de Colombia (1984-1999). Borradores de Investigación No. 13. Recuperado de http:// repository.urosario.edu.co/bitstream/handle/10336/11282/2593.pdf

Becker, G. (1957). The Economics of Discrimination. Chicago, Estados Unidos: University of Chicago Press.

Bernat, L. (2005). Análisis de género de las diferencias salariales en las siete principales áreas metropolitanas colombianas: ¿evidencia de discriminación? En Cuadernos PNUD-DANE: Investigaciones sobre género y desarrollo (pp. 65-96). Bogotá D.C., Colombia: PNUD.

Bernat, L. (2007). ¿Quiénes son las mujeres discriminadas?: enfoque distributivo de las diferencias salariales por género. Borradores de Economía y Finanzas No. 13. Universidad ICESI. Recuperado de https://www.icesi.edu.co/departamentos/economia/.../borrador_economia_0l3.pdf

Chávez, N. y Ríos, H. (2014). Discriminación salarial por género "efecto techo de cristal". Caso: siete áreas metropolitanas de Colombia. Revista Dimensión Empresarial, 12(2), 29-45. 
Collins, R. (1989). La Sociedad Credencialista. Sociología Histórica de la Educación y Estratificación. Madrid, España: AKAL.

Córtes, A. y Flórez, M. (2016). Diferencias salariales por género en el departamento de Santander, Colombia. Apuntes del CENES, 35(61), 267-302.

Duncan, O. y Duncan, B. (1955). A methodological analysis of segregation indexes. American Sociological Review, 20(2), 210-217. Recuperado de https://www.jstor.org/stable/2088328

Galvis, L. (2010). Diferenciales salariales por género y región en Colombia: Una aproximación con regresión por cuantíeles. Documentos de Trabajo sobre Economía Regional $\mathrm{N}^{\circ} 131$. Recuperado de http://www.banrep.gov.co/sites/default/files/publicaciones/archivos/DTSER-131.pdf

Hoyos, A., Ñopo, H. y Peña, X. (2010). The Persistent Gender Earnings Gap in Colombia, 1994-2006. Recuperado de http://repec.iza.org/dp5073.pdf

Iregui, A., Melo, L. y Ramírez, M. (2011). Diferenciales salariales en el mercado de trabajo formal en Colombia: evidencia a partir de una encuesta a nivel de firma. Reportes del Emisor 140. Recuperado de https://publicaciones.banrepcultural.org/index.php/emisor/article/view/7849/8229

Jarman, J., Blackburn, R. y Racko, G. (2012). The Dimensions of Occupational Gender Segregation in Industrial Countries. Sociology, 46(6), 1003-1019. Recuperado de http://www.jstor.org.bd.univalle. edu.co/stable/43497336

Karmel, T. y Maclachlan, M. (1988). Occupational Sex Segregation: Increasing or Decreasing? Economic Record, 64(3), 187-195.

Kramar, R. (1983). Economic Theory and the Sexual Segregation of the Labour Market. The Australian Quarterly, 55(4), 388-404. https://doi.org/10.2307/20635245

Nicolás, C., López, M. y Riquelme, P. (2009). La segregación ocupacional entre hombres y mujeres: teorías explicativas y análisis de su evolución reciente en España. Proyecto Social, 13, 38-62. Recuperado de http://hdl.handle.net/10201/21985

OIT -Organización Internacional del Trabajo-. (2013). Igualdad Salarial: Guía Introductoria. Recuperado de http://www.ilo.org/mexico/publicaciones/WCMS_226246/lang--es/index.htm

Pérez, A. (2016). Efecto de la Tenencia de Hijos en la Participación Laboral Femenina en Colombia, 2008, 2011 y 2015 (tesis de maestría). Universidad del Valle, Cali, Colombia.

Phelps, E. (1972). The Statistical Theory of Racism and Sexism. The American Economic Review, 62(4), 659-661. Recuperado de http://www.jstor.org/stable/1806107

Piñeros, L. (2009). Las Uniones Maritales, los Diferenciales Salariales y la Brecha Educativa en Colombia. Desarrollo y Sociedad, (64), 55-84.

Rodgers, W. (2006). Male White-Black Age Gaps, 1979-1994: A Distributional Analysis. Southern Economic Journal, 72(4), 773-793. 
Tenjo, J. (1993). 1976-1989: Cambios en los diferenciales salariales entre hombres y mujeres. Planeación \& Desarrollo, 24, 117-132.

Tenjo, J. y Herrera, P. (2009). Dos Ensayos sobre Discriminación: Discriminación salarial y discriminación en acceso al empleo por origen étnico y por género. Bogotá, Colombia: Pontificia Universidad Javeriana.

Wellington, A. (1993). Changes in the Male/Female Wage Gap, 1976-85. The Journal of Human Resources, 28(2), 383-411.

Wellington, A. (1994). Accounting for the Male/Female Wage Gap Among Whites: 1976 and 1985. American Sociological Review, 59(6), 839-848. 